\title{
Multi-cluster complexes
}

\author{
Cesar Ceballos $\|^{\dagger} \quad$ Jean-Philippe Labbé $\|^{*} \quad$ Christian Stump ${ }^{2} \|^{\beta}$ \\ ${ }^{1}$ Institut für Mathematik, Freie Universität Berlin, Germany \\ ${ }^{2}$ Institut für Algebra, Zahlentheorie, Diskrete Mathematik, Universität Hannover, Germany
}

\begin{abstract}
We present a family of simplicial complexes called multi-cluster complexes. These complexes generalize the concept of cluster complexes, and extend the notion of multi-associahedra of types $A$ and $B$ to general finite Coxeter groups. We study combinatorial and geometric properties of these objects and, in particular, provide a simple combinatorial description of the compatibility relation among the set of almost positive roots in the cluster complex.

Résumé. Nous présentons une famille de complexes simpliciaux appelés complexes des multi-amas. Ces complexes généralisent le concept de complexes des amas et étendent la notion de multi-associaèdre de type $A$ et $B$ aux groupes de Coxeter finis. Nous étudions des propriétés combinatoires et géométriques de ces objets et, en particulier nous fournissons une description combinatoire simple de la relation de compatibilité sur l'ensemble des racines presque positives du complexe des amas.
\end{abstract}

Keywords: Subword complexes, cluster complexes, generalized associahedra, multi-triangulations, Coxeter-Catalan combinatorics, sorting words

\section{Introduction}

Cluster complexes were introduced by S. Fomin and A. Zelevinsky to encode exchange graphs of cluster algebras [FZ03]. N. Reading then showed that the definition of cluster complexes of finite types can be extended to all finite Coxeter groups [Rea07a, Rea07b]. Recently, we presented a new combinatorial description of cluster complexes of finite types using subword complexes [CLS11]. These were introduced by A. Knutson and E. Miller, first in type $A$ to study the combinatorics of determinantal ideals and Schubert polynomials [KM05], and then for all Coxeter groups in [KM04]. We provide, for any finite Coxeter group $W$ and any Coxeter element $c \in W$, a subword complex which is isomorphic to the $c$-cluster complex of the corresponding type, and we thus obtain an explicit type-free characterization of $c$-clusters. In crystallographic types, this characterization is equivalent to a description obtained by K. Igusa and R. Schiffler in the context of cluster categories using algebraic techniques [IS10]. The present approach allows us to define a new family of simplicial complexes. These simplicial complexes have an additional parameter $k$, and they are, for $k=1$, isomorphic to $c$-cluster complexes. Therefore, we call the elements of this family multi-cluster complexes. They are different from generalized cluster complexes as defined by S. Fomin and N. Reading in [FR05]; in the generalized cluster complex, the vertices are given by the simple negative roots together with several distinguished copies of the positive roots, while the vertices

\footnotetext{
†Supported by DFG via the Research Training Group "Methods for Discrete Structures" and the Berlin Mathematical School.

¥Supported by a FQRNT Doctoral scholarship.

§Partially supported by a CRM-ISM fellowship, and by the DFG via the Research Group "Methods for Discrete Structures". 
of the multi-cluster complex correspond to the positive roots together with several distinguished copies of the simple negative roots. The multi-cluster complex is defined using the notion of sorting words introduced by N. Reading in [Rea07a]. In this text, we present the multi-cluster complex, related results and open problems contained in [CLS11]. In type $A$, the multi-cluster complex is isomorphic to the simplicial complex of multi-triangulations of a convex polygon [PP10, Stu11]. In type $B$, we showed that the multi-cluster complex is isomorphic to the simplicial complex of centrally symmetric multi-triangulations of a regular convex polygon. This result implies that the latter is a vertex-decomposable simplicial sphere. Multi-cluster complexes extend the concept of multi-associahedra to general finite Coxeter groups, and they unify several questions about polytopality - namely of spherical subword complexes, and of multiassociahedra of type $A$ and of type $B$ - in terms of polytopality of multi-cluster complexes. They also raise the question of finding a type-free definition of multi-Catalan numbers counting the number of facets of multi-cluster complexes, and of finding a family of simplicial complexes including multi-cluster complexes and generalized cluster complexes simultaneously.

Throughout the text, $(W, S)$ denotes a finite Coxeter system of rank $n$, and $c$ denotes a Coxeter element, i.e., the product of the generators in $S$ in some order. First, we adopt some writing conventions ; in order to emphasize the distinction between words and group elements, we write a word in the alphabet $S$ as a sequence between brackets $\left(a_{1}, a_{2}, \ldots, a_{k}\right)$ and use square letters such as $\mathbf{w}$ to denote them, and we write a group element as a concatenation of letters $a_{1} a_{2} \cdots a_{k}$ using normal script such as $w$ to denote them.

\section{Preliminaries}

In this section, we review the essential notions concerning multi-triangulations, subword complexes and cluster complexes of finite type.

\subsection{Multi-triangulations}

Let $\Delta_{m}$ be the simplicial complex with vertices being diagonals of a convex $m$-gon and faces being subsets of non-crossing diagonals. Its facets correspond to triangulations (i.e., maximal subsets of diagonals which are mutually non-crossing). This simplicial complex is the boundary complex of the dual associahedron, see [Hai84, Lee89]. It can be generalized using a positive integer $k$ with $2 k<m$ : define a $(k+1)$-crossing to be a set of $k+1$ diagonals which are pairwise crossing. A diagonal is called $k$-relevant if it is contained in some $(k+1)$-crossing, that is, if there are at least $k$ vertices of the $m$-gon on each side of the diagonal. The complex $\Delta_{m, k}$ is the simplicial complex of $(k+1)$-crossing free sets of $k$-relevant diagonals. Its facets are given by $k$-triangulations (i.e., maximal subsets of diagonals which do not contain a $(k+1)$-crossing). The reason for restricting the set of diagonals is that including all other diagonals would yield the join of $\Delta_{m, k}$ and an $m k$-simplex. This simplicial complex has been studied by several authors, see e.g. [DKM03, Jon05, JW07, Kra06, Nak00, Rub11, Stu11] ; an interesting recent treatment of $k$-triangulations can be found in [PS09].

In [Stu11], the following description of $\Delta_{m, k}$ is exhibited : let $\mathcal{S}_{n+1}$ be the symmetric group generated by the $n$ simple transpositions $s_{i}=(i i+1)$ for $1 \leq i \leq n$, where $n=m-2 k-1$. The $k$-relevant diagonals of a convex $m$-gon are in bijection with (positions of) letters in the word

$$
Q=(\underbrace{s_{n}, \ldots, s_{1}, \quad \cdots \quad s_{n}, \ldots, s_{1}}_{k \text { times } s_{n}, \ldots, s_{1}}, \quad s_{n}, \ldots, s_{1}, \quad s_{n}, \ldots, s_{2}, \quad \cdots \quad s_{n}, s_{n-1}, \quad s_{n})
$$


of length $k n+\left(\begin{array}{c}n+1 \\ 2\end{array}\right)=\left(\begin{array}{c}m \\ 2\end{array}\right)-m k$. If the vertices of the $m$-gon are cyclically labelled by the integers from 1 to $m$, the bijection sends the $i$-th letter of $Q$ to the $i$-th diagonal in lexicographic order. Under this bijection, a collection of diagonals forms a $k$-triangulation if and only if the complement of the corresponding subword in $Q$ forms a reduced expression for the permutation $[n+1, \ldots, 2,1] \in \mathcal{S}_{n+1}$. A similar approach which admits various possibilities for the word $Q$ was described in [PP10] in the context of sorting networks.

Example 2.1 For $m=5$ and $k=1$, we get $Q=\left(q_{1}, q_{2}, q_{3}, q_{4}, q_{5}\right)=\left(s_{2}, s_{1}, s_{2}, s_{1}, s_{2}\right)$. By labeling the vertices of the pentagon with the integers $\{1, \ldots, 5\}$ cyclically, the bijection sends the (position of the) letter $q_{i}$ to the $i$-th entry of the list of ordered diagonals $[13,14,24,25,35]$. On one hand, two cyclically consecutive diagonals in the list form a triangulation of the pentagon. On the other hand, the complement of two cyclically consecutive letters of $Q$ form a reduced expression for $[3,2,1]=s_{1} s_{2} s_{1}=s_{2} s_{1} s_{2} \in \mathcal{S}_{3}$.

The main objective is to describe and study a natural generalization of multi-triangulations to finite Coxeter groups.

\subsection{Subword complexes}

Let $Q=\left(q_{1}, \ldots, q_{r}\right)$ be a word in the generators $S$ of $W$ and let $\pi \in W$. The subword complex $\Delta(Q, \pi)$ was introduced by A. Knutson and E. Miller in order to study Gröbner geometry of Schubert varieties, see [KM05, Definition 1.8.1], and was further studied in [KM04]. It is defined as the simplicial complex whose faces are given by subwords $P$ of $Q$ for which the complement $Q \backslash P$ contains a reduced expression of $\pi$. Note that subwords come with their embedding into $Q$; two subwords $P$ and $P^{\prime}$ representing the same word are considered to be different if they involve generators at different positions within $Q$. In Example 2.1, we have seen an instance of a subword complex with $Q=\left(s_{2}, s_{1}, s_{2}, s_{1}, s_{2}\right)$ and $\pi=s_{1} s_{2} s_{1}=s_{2} s_{1} s_{2}$. In this case, $\Delta(Q, \pi)$ has vertices $\left\{q_{1}, \ldots, q_{5}\right\}$ and facets

$$
\left\{q_{1}, q_{2}\right\},\left\{q_{2}, q_{3}\right\},\left\{q_{3}, q_{4}\right\},\left\{q_{4}, q_{5}\right\},\left\{q_{5}, q_{1}\right\} .
$$

Let $Q^{\prime}$ be the word obtained by adding $s \in S$ at the end of a word $Q$. The Demazure product $\delta\left(Q^{\prime}\right)$ is recursively defined by

$$
\delta\left(Q^{\prime}\right)= \begin{cases}\pi s & \text { if } \ell(\pi s)>\ell(\pi) \\ \pi & \text { if } \ell(\pi s)<\ell(\pi),\end{cases}
$$

where $\pi=\delta(Q)$ is the Demazure product of $Q$, and where the Demazure product of the empty word is defined to be the identity element in $W$. Here, $\ell$ denotes the length function on $W$. It was shown in [KM04, Theorem 2.5] that subword complexes are vertex-decomposable. Moreover, a subword complex $\Delta(Q, \pi)$ is a sphere if and only if $\delta(Q)=\pi$, and a ball otherwise [KM04, Corollary 3.8].

\subsection{Cluster complexes}

In [FZ03], S. Fomin and A. Zelevinsky introduced the cluster complex associated to any crystallographic root system. This simplicial complex along with the generalized associahedron has become the object of intensive studies and generalizations in various contexts in mathematics, see for instance [CFZ02, MRZ03, Rea07a, HLT11]. A generator $s \in S$ is called initial (respectively final) in a Coxeter element $c$ if $\ell(s c)<\ell(c)$ (resp. $\ell(c s)<\ell(c)$ ). The group $W$ acts naturally on the real vector space $V$ with basis $\Delta=\left\{\alpha_{s}: s \in S\right\}$, its elements are called simple roots. Let $\Delta \subseteq \Phi^{+} \subseteq \Phi \subset V$ be the set of positive 
roots and the set of roots for $(W, S)$, respectively. Furthermore, let $\Phi_{\geq-1}=\Phi^{+} \cup-\Delta$ be the set of almost positive roots. By convention, we denote the maximal standard parabolic subgroup generated by $S \backslash\{s\}$ by $W_{\langle s\rangle}$, and the associated subroot system by $\Phi_{\langle s\rangle}$. For $s \in S$, the involution $\sigma_{s}: \Phi_{\geq-1} \longrightarrow \Phi_{\geq-1}$ is given by

$$
\sigma_{s}(\beta)= \begin{cases}\beta & \text { if }-\beta \in \Delta \backslash\left\{\alpha_{s}\right\} \\ s(\beta) & \text { otherwise. }\end{cases}
$$

In finite types, $c$-cluster complexes can be defined using a family $\|_{c}$ of $c$-compatibility relations on $\Phi_{\geq-1}$, see [RS11, Section 5]. This family $\|_{c}$ is characterized by the following two properties :

(i) for $s \in S$ and $\beta \in \Phi_{\geq-1}$,

$$
-\alpha_{s} \|_{c} \beta \Leftrightarrow \beta \in\left(\Phi_{\langle s\rangle}\right)_{\geq-1}
$$

(ii) for $\beta_{1}, \beta_{2} \in \Phi_{\geq-1}$ and $s$ being initial in $c$,

$$
\beta_{1}\left\|_{c} \beta_{2} \Leftrightarrow \sigma_{s}\left(\beta_{1}\right)\right\|_{s c s} \sigma_{s}\left(\beta_{2}\right)
$$

A maximal subset of pairwise $c$-compatible almost positive roots is called $c$-cluster. The $c$-cluster complex is the simplicial complex whose vertices are the almost positive roots and whose facets are $c$-clusters. It turns out that all $c$-cluster complexes for the various Coxeter elements are isomorphic, see [ Rea07a, Proposition 7.2] and [MRZ03, Propositions 3.4 and 4.10 and Section 1]. In crystallographic types, they are moreover isomorphic to the cluster complex as defined in [FZ03]. In particular, they are pure of dimension $n-1$, see [FZ03, Theorem 1.8] for crystallographic types and [Rea07a] for finite Coxeter groups in general.

\section{The multi-cluster complex}

We are now in the position to present the results and to define the central object, the multi-cluster complex. Let $\mathbf{c}=\left(c_{1}, \ldots, c_{n}\right)$ be the word corresponding to a Coxeter element $c \in W$, and let $\mathbf{w}_{\circ}(c)=$ $\left(w_{1}, \ldots, w_{N}\right)$ be the lexicographically first subword of $\mathbf{c}^{\infty}$ which represents a reduced expression for the longest element $w_{\circ} \in W$. The first theorem gives a description of the cluster complex as a subword complex.

Theorem 3.1 The subword complex $\Delta\left(\mathbf{c w}_{\circ}(c), w_{\circ}\right)$ is isomorphic to the c-cluster complex. The isomorphism is given by sending the letter $c_{i}$ of $\mathbf{c}$ to the negative root $-\alpha_{c_{i}}$, and the letter $w_{i}$ of $\mathbf{w}_{\circ}(c)$ to the positive root $w_{1} \cdots w_{i-1}\left(\alpha_{w_{i}}\right)$.

As an equivalent statement, we obtain the following explicit description of the $c$-compatibility relation.

Corollary 3.2 A subset $C$ of $\Phi_{\geq-1}$ is a c-cluster if and only if the complement of the corresponding subword in $\mathbf{c w}_{\circ}(c)=\left(c_{1}, \ldots, c_{n}, w_{1}, \ldots, w_{N}\right)$ represents a reduced expression for $w_{\circ}$.

Remark 3.3 This description was obtained by K. Igusa and R. Schiffler [IS10] for finite crystallographic Coxeter groups in the context of cluster categories using algebraic techniques. The present approach holds uniformly for all finite Coxeter groups, and is developed purely in the context of Coxeter group theory. 
Example 3.4 Let $W$ be the Coxeter group of type $B$ generated by $S=\left\{s_{1}, s_{2}\right\}$ and let $c=c_{1} c_{2}=s_{1} s_{2}$. Then the word $\mathbf{c w}_{\circ}(c)$ is given by $\left(c_{1}, c_{2}, w_{1}, w_{2}, w_{3}, w_{4}\right)=\left(s_{1}, s_{2}, s_{1}, s_{2}, s_{1}, s_{2}\right)$. The corresponding list of almost positive roots is

$$
\left[-\alpha_{1},-\alpha_{2}, \alpha_{1}, \alpha_{1}+\alpha_{2}, \alpha_{1}+2 \alpha_{2}, \alpha_{2}\right]
$$

The subword complex $\Delta\left(\mathbf{c w}_{\circ}(c), w_{\circ}\right)$ is an hexagon with facets being any two cyclically consecutive letters. The corresponding $c$-clusters are

$$
\left\{-\alpha_{1},-\alpha_{2}\right\},\left\{-\alpha_{2}, \alpha_{1}\right\},\left\{\alpha_{1}, \alpha_{1}+\alpha_{2}\right\},\left\{\alpha_{1}+\alpha_{2}, \alpha_{1}+2 \alpha_{2}\right\},\left\{\alpha_{1}+2 \alpha_{2}, \alpha_{2}\right\},\left\{\alpha_{2},-\alpha_{1}\right\} .
$$

Inspired by results in [Stu11] and [PP10], we generalize the subword complex in Theorem 3.1 by considering any Coxeter element word $\mathbf{c}$ to a power $k \in \mathbb{N}$. In type $A$, this generalization coincides with the description of the complex $\Delta_{m, k}$ given in [PP10].

Definition 3.5 The multi-cluster complex $\Delta_{c}^{k}(W)$ is the subword complex $\Delta\left(\mathbf{c}^{k} \mathbf{w}_{\circ}(c), w_{\circ}\right)$.

The multi-cluster complex is a vertex-decomposable sphere of dimension $k n-1$. The first interesting property of the multi-cluster complex is that it is independent of the Coxeter element $c$.

Theorem 3.6 All multi-cluster complexes $\Delta_{c}^{k}(W)$ for the various Coxeter elements are isomorphic.

We have seen in Section 2.1 that the multi-cluster complex of type $A_{m-2 k-1}$ is isomorphic to the simplicial complex whose facets correspond to $k$-triangulations of a convex $m$-gon,

$$
\Delta_{c}^{k}\left(A_{m-2 k-1}\right) \cong \Delta_{m, k}
$$

The simplicial complex of centrally symmetric $k$-triangulations of a regular $2 m$-gon (or $k$-triangulations of type $B$ ) was studied in algebraic and combinatorial contexts, see [SW09, RS10]. This simplicial complex is isomorphic to the multi-cluster complex of type $B_{m-k}$.

Theorem 3.7 The multi-cluster complex $\Delta_{c}^{k}\left(B_{m-k}\right)$ is isomorphic to the simplicial complex of centrally symmetric $k$-triangulations of a regular $2 m$-gon.

Using algebraic techniques, D. Soll and V. Welker proved that this simplicial complex is a (mod 2)homology-sphere [SW09, Theorem 10]. The previous theorem implies the following stronger result.

Corollary 3.8 The simplicial complex of centrally symmetric $k$-triangulations of a regular $2 m$-gon is a vertex-decomposable simplicial sphere of dimension $k(m-k)-1$.

This result together with the proof of [SW09, Conjecture 13] given in [RS10] $]^{(i)}$ implies the following conjecture by Soll and Welker.

Corollary 3.9 ([SW09, Conjecture 17]) For the term-order $\preceq$ defined in [SW09] Section 7], the initial ideal $\operatorname{in}_{\preceq}\left(I_{n, k}\right)$ of the determinantal ideal $I_{n, k}$ defined in [SW09. Section 3] is spherical.

The last result describes all spherical subword complexes in terms of faces of multi-cluster complexes.

Theorem 3.10 A simplicial sphere can be realized as a subword complex of a given finite type $W$ if and only if it is the link of a face of a multi-cluster complex $\Delta_{c}^{k}(W)$.

(i). The proof appeared in Section 7 in the arxiv version, see http://arxiv. org/abs/0904.1097v2. 
Corollary 3.11 The following two statements are equivalent.

(i) Every spherical subword complex is polytopal.

(ii) Every multi-cluster complex is polytopal.

\section{Open problems}

In this last section, we discuss open problems on multi-cluster complexes.

Open Problem 4.1 Find multi-Catalan numbers counting the number of facets in the multi-cluster complex.

Although a formula in terms of invariants of the group for the number of facets of the generalized cluster complex defined by S. Fomin and N. Reading is known [FR05, Proposition 8.4], a general formula in terms of invariants of the group for the multi-cluster complex is yet to be found. An explicit formula for type $A$ can be found in [Jon05, Corollary 17]. In type $B$, a formula was conjectured in [SW09, Conjecture 13] and proved in [RS10] ${ }^{[\text {(ii) }}$. In type $I_{2}(m)$, the number of facets of the multi-cluster complex is equal to the number of facets of a $2 k$-dimensional cyclic polytope on $2 k+m$ vertices. These three formulas can be reformulated in terms of invariants of the Coxeter groups of type $A, B$ and $I_{2}$ as follows,

$$
\prod_{0 \leq j<k} \prod_{1 \leq i \leq n} \frac{d_{i}+h+2 j}{d_{i}+2 j},
$$

where $d_{1} \leq \ldots \leq d_{n}$ are the degrees of the corresponding group, and $h$ is its Coxeter number. In general, this product is not an integer. The smallest example we are aware of is type $D_{6}$ with $k=5$. Thus, this product cannot count facets of the multi-cluster complex in general. Observe that this counting formulas in types $A, B$ and $I_{2}$ can naturally be enriched with the parameter $m$ such that it reduces for $k=1$ to the Fuss-Catalan numbers counting the number of facets in the generalized cluster complexes. The next open problem raises the question of finding a family of simplicial complexes that includes the generalized cluster complexes of S. Fomin and N. Reading [FR05] and the multi-cluster complexes.

Open Problem 4.2 Find a family of simplicial complexes which simultaneously contains the generalized cluster complexes and the multi-cluster complexes.

The next problem extends the open problem of finding the diameter of the associahedron to the family of multi-cluster complexes.

Open Problem 4.3 Find the diameter of the facet-adjacency graph of the multi-cluster complex $\Delta_{c}^{k}(W)$.

In view of Corollary 3.11, the next conjecture restricts the study of [KM04, Question 6.4].

Conjecture 4.4 The multi-cluster complex is the boundary complex of a simplicial polytope.

This conjecture holds for $k=1$; the multi-cluster complex in this case is the dual of the polytopal realization of $c$-generalized associahedra constructed in [HLT11]. In types $A$ and $B$, this conjecture coincides with the conjectures on polytopality of multi-associahedra, see [Jon05][SW09]. Using Gale's evenness condition, it can be shown in type $I_{2}(m)$, that the multi-cluster complex $\Delta_{c}^{k}\left(I_{2}(m)\right)$ is isomorphic to the boundary complex of a $2 k$-dimensional cyclic polytope on $2 k+m$ vertices. This last example shows that the conjecture also holds for dihedral groups.

(ii). The proof appeared in Section 7 in the arxiv version, see http://arxiv. org/abs/0904.1097v2. 


\section{Acknowledgments}

The authors would like to thank Drew Armstrong, Christophe Hohlweg, Carsten Lange, Emerson Leon, Ezra Miller, Vincent Pilaud, Luis Serrano, Hugh Thomas, and Günter Ziegler for fruitful discussions.

\section{Références}

[CFZ02] Frédéric Chapoton, Sergey Fomin, and Andrei Zelevinsky, Polytopal realizations of generalized associahedra, Canad. Math. Bull. 45 (2002), no. 4, 537-566.

[CLS11] Cesar Ceballos, Jean-Philippe Labbé, and Christian Stump, Subword complexes, cluster complexes, and generalized multi-associahedra, preprint (2011), available at arXiv:abs/1108.1776.

[DKM03] Andreas Dress, Jack H. Koolen, and Vincent Moulton, On line arrangements in the hyperbolic plane, Eur. J. Comb. 23 (2003), no. 5, 549-557.

[FR05] Sergey Fomin and Nathan Reading, Generalized cluster complexes and Coxeter combinatorics, Int. Math. Res. Notices 2005 (2005), no. 44, 2709-2757.

[FZ03] Sergey Fomin and Andrei Zelevinsky, Y-Systems and generalized associahedra, Ann. Math. 158 (2003), no. 3, 977-1018.

[Hai84] Mark Haiman, Constructing the associahedron, unpublished (1984).

[HLT11] Christophe Hohlweg, Carsten E.M.C. Lange, and Hugh Thomas, Permutahedra and generalized associahedra, Adv. Math. 226 (2011), no. 1, 608-640.

[IS10] Kiyoshi Igusa and Ralf Schiffler, Exceptional sequences and clusters, J. Algebra 323 (2010), no. 8, 2183-2202.

[Jon05] Jakob Jonsson, Generalized triangulations and diagonal-free subsets of stack polyominoes, J. Comb. Theory, Ser. A 112 (2005), no. 1, 117-142.

[JW07] Jakob Jonsson and Volkmar Welker, A spherical initial ideal for Pfaffians, Ill. J. Math. 51 (2007), no. 4, 1397-1407.

[KM04] Allen Knutson and Ezra Miller, Subword complexes in Coxeter groups, Adv. Math. 184 (2004), no. $1,161-176$.

[KM05] Allen Knutson and Ezra Miller, Gröbner geometry of Schubert polynomials, Ann. Math. (2) 161 (2005), no. 3, 1245-1318.

[Kra06] Christian Krattenthaler, Growth diagrams, and increasing and decreasing chains in fillings of Ferrers shapes, Adv. Appl. Math. 37 (2006), no. 3, 404-431.

[Lee89] Carl W. Lee, The associahedron and triangulations of the n-gon, European J. Combin. 10 (1989), no. 6, 551-560.

[MRZ03] Robert Marsh, Markus Reineke, and Andrei Zelevinsky, Generalized associahedra via quiver representations, Trans. Amer. Math. Soc. 355 (2003), no. 10, 4171-4186.

[Nak00] Tomoki Nakamigawa, A generalization of diagonal flips in a convex polygon, Theor. Comput. Sci. 235 (2000), no. 2, 271-282.

[PP10] Vincent Pilaud and Michel Pocchiola, Multitriangulations, pseudotriangulations and primitive sorting networks, preprint (2010), available at arXiv:abs/1009.5344. 
[PS09] Vincent Pilaud and Francisco Santos, Multitriangulations as complexes of star polygons, Discrete Comput. Geom. 41 (2009), no. 2, 284-317.

[Rea07a] Nathan Reading, Clusters, Coxeter-sortable elements and noncrossing partitions, Trans. Amer. Math. Soc. 359 (2007), no. 12, 5931-5958.

[Rea07b] Sortable elements and Cambrian lattices, Algebra universalis 56 (2007), no. 3-4, 411-437.

[RS10] Martin Rubey and Christian Stump, Crossings and nestings in set partitions of classical types, Electron. J. Combin. 17 (2010), no. 1, R120.

[RS11] Nathan Reading and David E. Speyer, Sortable elements in infinite Coxeter groups, Trans. Amer. Math. Soc. 363 (2011), no. 2, 699-761.

[Rub11] Martin Rubey, Increasing and decreasing sequences in fillings of moon polyominoes, Adv. Appl. Math. 47 (2011), no. 1, 57-87.

[Stu11] Christian Stump, A new perspective on k-triangulations, J. Comb. Theory, Ser. A 118 (2011), no. $6,1794-1800$.

[SW09] Daniel Soll and Volkmar Welker, Type-B generalized triangulations and determinantal ideals, Discrete Math. 309 (2009), no. 9, 2782-2797. 